ANNALES

POLONICI MATHEMATICI

$84.1(2004)$

\title{
On time reparametrizations and isomorphisms of impulsive dynamical systems
}

\author{
by Krzysztof Ciesielski (Kraków)
}

\begin{abstract}
We prove that for a given impulsive dynamical system there exists an isomorphism of the basic dynamical system such that in the new system equipped with the same impulse function each impulsive trajectory is global, i.e. the resulting dynamics is defined for all positive times. We also prove that for a given impulsive system it is possible to change the topology in the phase space so that we may consider the system as a semidynamical system (without impulses).
\end{abstract}

0. Introduction. The problem of isomorphisms of dynamical systems is of fundamental importance. In isomorphisms we may reparametrize time, transform homeomorphically the phase space or do both. Several results on isomorphisms of dynamical systems are known, in particular (under suitable assumptions) one can treat a local system as a global one ([Ca]).

In an impulsive dynamical system, impulsive trajectories with an infinite number of impulses (discontinuities) give much of the flavour of impulsive systems. For such a trajectory, the motion of a point $x$ is defined in an interval $\left[0, \alpha_{x}\right)$, where $\alpha_{x}$ need not be $+\infty$. From the point of view of impulsive systems, the trajectories with an infinite number of impulses and with domain $[0,+\infty)$ are of special interest (cf. for instance [K1], [K3], [K4], [LBS]).

In this paper, we show that under some natural assumptions we can find a time reparametrization of the dynamical system which is a basic system for the impulsive system under investigation (and, consequently, an isomorphism of that system) so that the resulting impulsive system satisfies the above condition, i.e. the impulsive motion is defined for all positive times, for any $x$. We do not change the impulse function and the space, the isomorphism only changes the "speed" on the trajectories of the original system.

2000 Mathematics Subject Classification: 37B99, 54H20, 34A37.

Key words and phrases: impulsive dynamical system, time reparametrization, isomorphism of dynamical systems, flow, section, impulse, metrizability, continuity, escape time. 
Simultaneously, we show that (under the same assumptions) it is possible to modify the topology in the phase space in such a way that the discontinuities in the impulse function disappear. So, instead of an impulsive dynamical system we obtain a semidynamical system where the phase space is the same, but equipped with another topology. We improve the structure of the system but we can get a "worse" topology. The new topology is the same as the original one in a small neighbourhood of each point which is neither an impulse point nor the image of one. This new topology is obtained as a quotient topology induced by an upper semicontinuous decomposition of the suitably modified phase space. We give a necessary and sufficient condition for metrizability of the new topology.

Analogous theorems also hold for impulsive systems given by semidynamical systems with global backward extensions.

To prove our theorems, we use some advanced results of general topology and some theorems from the theory of dynamical systems. Impulsive systems are defined on metric spaces. On the basis of a given system, the impulse set and the properties of sections we "cut" the phase space and "glue" it in a suitable way to construct a new phase space, using, in particular, metrization theorems and upper semicontinuous decompositions. The resulting topological space need not be metrizable. However, it satisfies the assumptions of Carlson's theorems on isomorphisms, so we may apply them to obtain a time reparametrization and then come back to the original metric space.

Along the way we prove that if two dynamical systems are isomorphic as semidynamical systems then they are also isomorphic as dynamical systems. We also show that if a semidynamical (or dynamical) system is isomorphic as a pseudo-system to another pseudo-system and this isomorphism satisfies some natural conditions (in particular, the continuity conditions required in the definition of non-pseudo-systems), then the other pseudo-system is a system (semidynamical or dynamical) and the isomorphism is an isomorphism of (non-pseudo) systems. These results are not connected with impulsive systems but they seem to be interesting for the general theory of dynamical systems.

1. Preliminaries. In a topological space $X$ we define a local semidynamical system (a local semiflow) $(X, \pi)$ if $\pi$ is a function with domain $\operatorname{dom}_{\pi} \subset \mathbb{R}_{+} \times X$ and with values in $X$ satisfying the following conditions:

- for any $x \in X$ there is an $\omega_{x} \in(0,+\infty]$ such that $\pi(t, x)$ is defined for $t \in\left[0, \omega_{x}\right)$ (the local existence axiom),

- $\pi(0, x)=x$ for any $x \in X$ (the initial value axiom),

- $\pi(t, \pi(s, x))=\pi(t+s, x)$ for all $t, s \in \mathbb{R}, x \in X$ (the semigroup axiom), 
- $\operatorname{dom}_{\pi}$ is open in $\mathbb{R}_{+} \times X$ (Kamke's axiom),

- $\pi$ is continuous (the continuity axiom).

Replacing $\mathbb{R}_{+}$by $\mathbb{R}$ and the intervals $\left[0, \omega_{x}\right)$ by $\left(\alpha_{x}, \omega_{x}\right)$ where $-\infty \leq$ $\alpha_{x}<0$ we get the definition of a local dynamical system (a local flow). If $\operatorname{dom}_{\pi}=\mathbb{R}_{+} \times X$ (resp. $\mathbb{R} \times X$ ) we speak about a semidynamical (resp. dynamical) system. Without the continuity axiom and Kamke's axiom we speak about a pseudo-dynamical (resp. pseudo-semidynamical) system (possibly local).

We define a positive trajectory of $x$ as $\pi^{+}(x)=\pi([0,+\infty) \times\{x\})$. A segment of the trajectory is the set $\pi(\Delta, x)$, where $\Delta$ is a bounded interval.

In a semidynamical system, we set $F(t, y)=\{z \in X: \pi(t, z)=y\}$ for $t \geq 0$ and $y \in X$. In an analogous way we define $F(\Delta, D)$ for $\Delta \subset[0,+\infty)$ and $D \subset X$. A point $x \in X$ is said to be a start point if $F(t, x)=\emptyset$ for all $t>0$. A function $\sigma:(\alpha, 0] \rightarrow X$ is called a left maximal solution through $x$ if $\sigma(0)=x, \pi(t, \sigma(s))=\sigma(t+s)$ whenever $s, t+s \in(\alpha, 0]$ and $t \geq 0$, and $\sigma$ is maximal (with respect to inclusion) with these properties. For a semidynamical system without start points, by the negative escape time of $x$ we mean $N(x)=\inf \{\alpha>0:(-\alpha, 0]$ is the domain of a left maximal solution through $x\}$. For this definition and some remarks on it, see $[\mathrm{MC}],[\mathrm{CO}],[\mathrm{C} 1]$. A semidynamical system is said to be a system with global backward extension if it has no start point and $N(x)=+\infty$ for any $x \in X$. Any dynamical system is a semidynamical system with global backward extension. For some comments on this kind of systems, see [E1], [E2], [EK].

A semidynamical system is said to be a system with negative uniqueness (or with negative unicity, see e.g. $[\mathrm{BH}]$ ) if $F(t, x)$ has at most one element for any $t>0$ and $x \in X$. It is easy to verify that for a semidynamical system with negative uniqueness we have $N(x)=\sup \{t \geq 0: F(t, x) \neq \emptyset\}$. In a semidynamical system $\left(X, \pi_{i}\right)$ we write $F_{i}(\cdot, \cdot)$ instead of $F(\cdot, \cdot)(i \in \mathbb{N})$.

For the elementary properties of dynamical systems and semidynamical systems, see [BH], [BS], [NS], [P1], [P2], [V].

Two local dynamical (or semidynamical) systems $\left(X, \pi_{1}\right)$ and $\left(X, \pi_{2}\right)$ are isomorphic if there exists a continuous function $\chi$ from $\operatorname{dom}_{\pi_{1}}$ to $\mathbb{R}$ (or $\mathbb{R}_{+}$) which satisfies the following conditions:

- $\chi(0, x)=0$ for any $x \in X$,

- $\pi_{1}(t, x)=\pi_{2}(\chi(t, x), x)$ for every $t, s \in \mathbb{R}\left(\right.$ or $\left.\mathbb{R}_{+}\right)$and $x \in X$,

- for any $x \in X$ the function $\chi_{x}=\chi(\cdot, x)$ is an increasing homeomorphism from $\left(\alpha_{x}^{1}, \omega_{x}^{1}\right)$ to $\left(\alpha_{x}^{2}, \omega_{x}^{2}\right)$ (or from $\left[0, \omega_{x}^{1}\right)$ to $\left[0, \omega_{x}^{2}\right)$ ), where $\alpha_{x}^{i}, \omega_{x}^{i}$ are as in the local existence axiom,

- $\chi$ is continuous.

The function $\chi$ is called a time reparametrization. 
REMARK. We may also consider more general isomorphisms, i.e. between systems on two phase spaces $X$ and $Y$ and involving also a homeomorphism between $X$ and $Y$. The definition presented above is a particular case of the general one, formulated in the case where we consider the systems on the same space and the homeomorphism equal to identity. Thus the results on the existence of such isomorphism give more, as we prove the existence of a system where we do not change the space, we have only a time reparametrization. All such results are certainly applicable also for more general isomorphisms. Some important existence results were obtained just for isomorphisms where we only reparametrize time (cf. [Ca], [MC]).

Let us recall one of the fundamental results of Carlson ([Ca]). We present it in the form which will be of use in this paper.

Theorem 0. Let $X$ be a topological space such that $[0,1] \times X$ is normal and let $\left(X, \pi_{0}\right)$ be a local dynamical (resp. semidynamical) system. Then there exists a global dynamical (resp. semidynamical) system $\left(X, \pi_{1}\right)$ isomorphic to $\left(X, \pi_{0}\right)$ such that the time reparametrization $\chi: \mathbb{R} \times X \rightarrow \mathbb{R}$ (resp. $\chi$ : $\left.\mathbb{R}_{+} \times X \rightarrow \mathbb{R}_{+}\right)$satisfies $|\chi(t, x)| \geq|t|($ resp. $\chi(t, x) \geq t)$ for all $(t, x) \in \operatorname{dom}_{\pi_{0}}$.

For more information on isomorphisms of dynamical systems, the reader is referred to $[\mathrm{AV}],[\mathrm{Ca}],[\mathrm{MC}],[\mathrm{U} 1]-[\mathrm{U} 3],[\mathrm{UE}]$.

An impulsive system $(X, \pi, M, I)$ consists of a semidynamical (possibly dynamical) system $(X, \pi)$ on a metric space together with a nonempty closed subset $M$ of $X$ and a continuous function $I: M \rightarrow X$. We assume that for each $x \in M$ there is an $\varepsilon_{x}>0$ such that $\pi\left(\left(-\varepsilon_{x}, 0\right), x\right) \cap M=\emptyset$ and $\pi\left(\left(0, \varepsilon_{x}\right), x\right) \cap M=\emptyset$ in the case of dynamical systems, and $F\left(\left(0, \varepsilon_{x}\right), x\right) \cap$ $M=\emptyset$ and $\pi\left(\left(0, \varepsilon_{x}\right), x\right) \cap M=\emptyset$ in the case of semidynamical systems. These conditions mean that the points of $M$ are isolated on every trajectory of the system $(X, \pi)$. We call $M$ the impulse set and $I$ the impulse function. We set $M^{\oplus}(x)=\left(\pi^{+}(x) \cap M\right) \backslash\{x\}$.

We define a function $\phi: X \rightarrow(0,+\infty]$ in the following way. If $M^{\oplus}(x)=\emptyset$ then $\phi(x)=+\infty$. If $M^{\oplus}(x) \neq \emptyset$ then $\phi(x)$ is defined as the number $s$ such that $\pi(t, x) \notin M$ for $t \in(0, s)$ and $\pi(s, x) \in M$ (i.e. $\phi(x)$ is the smallest positive time for which the positive trajectory of $x$ meets $M)$. For $x \in M$ we call the point $\pi(\phi(x), x)$ the impulse point of $x$.

The trajectory $\widetilde{\pi}^{+}(x)$ of a point $x$ is defined as follows. We start from $x$. If $M^{\oplus}(x)=\emptyset$ then we put $\widetilde{\pi}(s, x)=\pi(s, x)$ for any $s \geq 0$. If $M^{\oplus}(x) \neq \emptyset$ then we put $\widetilde{\pi}(s, x)=\pi(s, x)$ for $s<\phi(x)$ and $\widetilde{\pi}(\phi(x), x)=I(\pi(\phi(x), x))$. Then we continue the procedure starting from $\tilde{\pi}(\phi(x), x)$ and so on.

For $x \in X$, we set

$$
\begin{array}{ll}
x^{1}=\pi(\phi(x), x), & x^{1+}=\tilde{\pi}(\phi(x), x)=I(\pi(\phi(x), x))=I\left(x^{1}\right), \\
x^{2}=\pi\left(\phi\left(x^{1+}\right), x^{1+}\right), & x^{2+}=\tilde{\pi}\left(\phi\left(x^{1+}\right), x^{1+}\right)=I\left(\pi\left(\phi\left(x^{1+}\right), x^{1+}\right)\right)=I\left(x^{2}\right)
\end{array}
$$


and so on. Thus, for any $x \in X$ exactly one of the following three conditions holds:

(i) $M^{\oplus}(x)=\emptyset$,

(ii) for some $n \geq 1: x^{k+}$ is defined for $k=1, \ldots, n$ and $M^{\oplus}\left(x^{n+}\right)=\emptyset$,

(iii) for any $k \geq 1$ : $x^{k+}$ is defined and $M^{\oplus}\left(x^{k+}\right) \neq \emptyset$.

For any $x \in X$ we define the escape time $\widetilde{\omega}(x)$ as $\sup \{s: \widetilde{\pi}(s, x)$ is defined $\}$.

Clearly, if $x$ satisfies (i) or (ii) then $\widetilde{\omega}(x)=+\infty$. If $x$ satisfies (iii) then either $\widetilde{\omega}(x)=+\infty$ or $\widetilde{\omega}(x) \in(0,+\infty)$.

In the impulsive system $\left(X, \pi_{i}, M, I\right)$ we denote the escape time of $x$ by $\widetilde{\omega}_{i}(x)(i \in \mathbb{N})$.

For more information on impulsive systems, see [C2], [C3], [K1]-[K4], [LBS].

For a dynamical system $(X, \pi)$ and $\lambda>0$ a set $S$ containing $x$ is called a section, or a $\lambda$-section, through $x$ if $U_{S}=\pi((-\lambda, \lambda), S)$ is a neighbourhood (not necessarily open) of $x$ and for every $y \in U$ there are unique $z \in S$ and unique $t \in(-\lambda, \lambda)$ with $\pi(t, z)=y$ (see for instance [BS], [NS], [C4]). The set $U_{S}$ is then called a tube (given by the section $S$ ) through $x$.

For a semidynamical system $(X, \pi)$ and $\lambda>0$ a closed set $S$ containing $x$ is called a section, or a $\lambda$-section, through $x$ if there exists a closed set $L$ such that $F(\lambda, L)=S, F([0,2 \lambda], L)$ is a neighbourhood (not necessarily open) of $x$ and $F(\mu, L) \cap F(\nu, L)=\emptyset$ for $0 \leq \mu<\nu \leq 2 \lambda$. We then call $F([0,2 \lambda], L)$ a tube, or $\lambda$-tube, and $L$ a bar. This agrees with the previous definition in the case of dynamical systems (cf. [C4]). In [C4] an existence theorem for sections is proved.

For the following conditions (TC) and (STC), assume we are given an impulsive system with impulse set $M$.

A tube $U_{S}$ given by a section $S$ through $x$ such that $S \subset M \cap U_{S}$ will be called a TC-tube through $x$. We will say that $x \in M$ satisfies (TC) (Tube Condition) if there exists a TC-tube through $x$.

A tube $U_{S}$ given by a section $S$ through $x$ such that $S=M \cap U_{S}$ will be called an STC-tube through $x$. We will say that a point $x \in M$ satisfies (STC) (Strong Tube Condition) if there exists an STC-tube through $x$.

For some comments concerning these definitions, see [C2] (cf. also [K1]).

In a metric space $X$, we denote by $B(x, \varepsilon)$ the open ball of radius $\varepsilon$ centred at $x$. In this paper a neighbourhood of $x$ need not be an open set. We say that two topologies $\tau_{1}, \tau_{2}$ on $X$ are locally identical at $x \in X$ if they have a common neighbourhood basis at $x$.

\section{Three lemmas}

Lemma 1. Let $\left(X, \pi_{0}\right)$ be a dynamical system and $\left(X, \pi_{1}\right)$ be a semidynamical system with negative uniqueness such that $N(x)=+\infty$ for any 
$x \in X$. Assume that $\chi: \mathbb{R}_{+} \times X \rightarrow \mathbb{R}_{+}$is a time reparametrization giving an isomorphism of $\left(X, \pi_{0}\right)$ and $\left(X, \pi_{1}\right)$ as semidynamical systems. Then $\left(X, \pi_{1}\right)$ is a dynamical system and $\left(X, \pi_{0}\right)$ and $\left(X, \pi_{1}\right)$ are isomorphic as dynamical systems. There exists a time reparametrization $\psi: \mathbb{R} \times X \rightarrow \mathbb{R}$ such that $\left.\psi\right|_{\mathbb{R}_{+} \times X}=\chi$.

From Lemma 1 we have an immediate

Corollary 1. Let $\left(X, \pi_{0}\right)$ and $\left(X, \pi_{1}\right)$ be dynamical systems. Assume that $\chi: \mathbb{R}_{+} \times X \rightarrow \mathbb{R}_{+}$is a time reparametrization giving an isomorphism of $\left(X, \pi_{0}\right)$ and $\left(X, \pi_{1}\right)$ as semidynamical systems. Then $\left(X, \pi_{0}\right)$ and $\left(X, \pi_{1}\right)$ are isomorphic as dynamical systems. There exists a time reparametrization $\psi: \mathbb{R} \times X \rightarrow \mathbb{R}$ such that $\left.\psi\right|_{\mathbb{R}_{+} \times X}=\chi$.

REMARK. Lemma 1 gives, in particular, a condition under which a semidynamical system with negative uniqueness gives a dynamical system. There are other such conditions: if $X$ is first countable and locally compact, the suitable theorem was proved in [C1]; if $X$ is a manifold, the result is due to O. Hajek (see $[\mathrm{Ha}])$.

Proof of Lemma 1. We have $\pi_{0}(t, x)=\pi_{1}(\chi(t, x), x), \chi(0, x)=0$ for $t \geq 0, x \in X$, and $\chi(\cdot, x): \mathbb{R}_{+} \rightarrow \mathbb{R}_{+}$is a homeomorphism for any $x$. Define

$$
\psi(t, x)= \begin{cases}-\chi\left(-t, \pi_{0}(t, x)\right) & \text { for } t \leq 0 \\ \chi(t, x) & \text { for } t \geq 0\end{cases}
$$

The continuity of $\psi$ and the condition $\left.\psi\right|_{\mathbb{R}_{+} \times X}=\chi$ are obvious. Now we show that $\pi_{0}(t, x)=\pi_{1}(\psi(t, x), x)$ for any $t$ and $x$. We need to verify this for $t<0$. Let $p=\pi_{1}\left(-\chi\left(-t, \pi_{0}(t, x)\right), x\right)$. Then $\pi_{1}\left(\chi\left(-t, \pi_{0}(t, x)\right), p\right)=x, x \in$ $\pi_{1}^{+}(x)=\pi_{0}^{+}(x)$ and $p=\pi_{0}(-s, x)$ for some $s \geq 0$. Set $\alpha=\chi\left(-t, \pi_{0}(t, x)\right)$. Then we have $\pi_{1}\left(\alpha, \pi_{0}(-s, x)\right)=x$. Moreover, $\pi_{1}\left(\chi\left(-t, \pi_{0}(t, x)\right), \pi_{0}(t, x)\right)=$ $\pi_{0}\left(-t, \pi_{0}(t, x)\right)=x$, so $\pi_{1}\left(\alpha, \pi_{0}(t, x)\right)=x$. Thus $p=\pi_{0}(-s, x)=\pi_{1}(-\alpha, x)$ $=\pi_{0}(t, x)$, which is our claim.

It remains to prove that $\psi(\cdot, x): \mathbb{R} \rightarrow \mathbb{R}$ is a homeomorphism for any $x$. We know that $\left.\psi(\cdot, x)\right|_{[0,+\infty)}$ is a homeomorphism from $\mathbb{R}_{+}$to $\mathbb{R}_{+}$.

We first show that $\left.\psi(\cdot, x)\right|_{(-\infty, 0]}$ is injective. Let $\psi(t, x)=\psi(s, x)$. Then $-\chi\left(-t, \pi_{0}(t, x)\right)=-\chi\left(-s, \pi_{0}(s, x)\right)$ and

$$
\pi_{0}(t, x)=\pi_{1}\left(-\chi\left(-t, \pi_{0}(t, x)\right), x\right)=\pi_{1}\left(-\chi\left(-s, \pi_{0}(s, x)\right), x\right)=\pi_{0}(s, x) .
$$

Consequently, $-\chi\left(-t, \pi_{0}(t, x)\right)=-\chi\left(-s, \pi_{0}(s, x)\right)$ as $-t,-s \geq 0$ and therefore $t=s$.

Since $\psi(0, x)=x$ we conclude that $\psi(\cdot, x)$ is an increasing bijection from $(-\infty, 0]$ to $(\iota, 0]$ for some $\iota$. Now we need only show that $\iota=-\infty$. Fix $\beta<0$ and $x \in X$. We have to prove that there exists a $u<0$ with $\psi(u, x)=\beta$.

There is a $z$ such that $\pi_{1}(\beta, x)=z$. 
First assume that $z$ is stationary in the system $\pi_{1}$, i.e. $\pi_{1}(v, z)=z$ for any $v \geq 0$. Then so is $x=\pi_{1}(-\beta, z)$. For $t<0$ we have $\psi(t, x)=$ $-\chi\left(-t, \pi_{0}(t, x)\right)=-\chi(-t, x)$, so $\psi([0,+\infty), x)=-\chi([0,+\infty), x)=(-\infty, 0]$.

Now suppose $z$ is non-stationary. Again, $x=\pi_{1}(-\beta, z) \in \pi_{1}^{+}(z)=\pi_{0}^{+}(z)$, so there is an $s \geq 0$ such that $x=\pi_{0}(s, z)=\pi_{1}(\chi(s, z), z)$.

In the case where $\pi_{1}(\cdot, z)$ is injective on $[0,+\infty)$ in view of the above reasoning we have $-\beta=\chi(s, z)$, so $\beta=-\chi(s, z)=-\chi\left(s, \pi_{0}(-s, x)\right)=$ $\psi(-s, x)$.

The last case to consider is when $z$ is periodic for $\pi_{1}$, i.e. there is a $T>0$ such that $\pi_{1}(T, z)=z$. Here $-\beta=\chi(s, z)+k T$ for some integer $k$. Then $\pi_{1}(\chi(s, z)+k T, z)=\pi_{1}(-\beta, z)$ and there exists a $v \geq 0$ with $\chi(v, z)=\chi(s, z)+k T=-\beta$ as $\chi(\cdot, z): \mathbb{R}_{+} \rightarrow \mathbb{R}_{+}$is a homeomorphism. Thus $\pi_{1}(\chi(v, z), z)=\pi_{1}(\chi(s, z)+k T, z)=\pi_{1}(\chi(s, z), z)$ and further $x=\pi_{0}(s, z)=\pi_{1}(\chi(s, z), z)=\pi_{1}(\chi(v, z), z)=\pi_{0}(v, z)$, which implies that $z=\pi_{0}(-v, x)$ as $\pi_{0}$ is a dynamical system. Finally, we have $\beta=-\chi(v, z)=-\chi\left(v, \pi_{0}(-v, x)\right)=\psi(-v, x)$. The proof is complete.

REMARK. Note that the time reparametrization $\chi$ obtained in Lemma 1 need not be unique. It is enough to consider the trivial example with onepoint space $\{x\}$. Then for a given $\chi: \mathbb{R}_{+} \rightarrow \mathbb{R}_{+}$any increasing homeomorphism $\psi: \mathbb{R} \rightarrow \mathbb{R}$ satisifes the required condition provided that $\left.\psi\right|_{\mathbb{R}_{+}}=\chi$.

Lemma 2. Let $\left(X, \pi_{0}\right)$ be a semidynamical system and $\left(X, \pi_{1}\right)$ a pseudosemidynamical system. Let $\chi: \mathbb{R}_{+} \times X \rightarrow \mathbb{R}_{+}$be as in the definition of an isomorphism of semidynamical systems. Assume also that $\chi(t, x) \geq t$ for any $t, x$. Then $\pi_{1}$ is continuous, i.e. $\left(X, \pi_{1}\right)$ is a semidynamical system, and it is isomorphic to $\left(X, \pi_{0}\right)$ as a semidynamical system.

Proof. Let $s_{n} \rightarrow s$ and $x_{n} \rightarrow x$. For each $n$ there exists a unique $t_{n}$ such that $s_{n}=\chi\left(t_{n}, x_{n}\right)$. We have $0 \leq t_{n} \leq s_{n}$, so $\left(t_{n}\right)$ is bounded as $\left(s_{n}\right)$ is convergent. We claim that $\left(t_{n}\right)$ is convergent. If not, there are two convergent subsequences $\left(t_{n_{k}}\right)$ and $\left(t_{n_{j}}\right)$ of $\left(t_{n}\right)$ with $t_{n_{k}} \rightarrow t^{\prime}$ and $t_{n_{j}} \rightarrow t^{\prime \prime}, t^{\prime} \neq t^{\prime \prime}$. However, $s_{n_{k}}=\chi\left(t_{n_{k}}, x_{n_{k}}\right) \rightarrow \chi\left(t^{\prime}, x\right)$ and $s_{n_{j}}=\chi\left(t_{n_{j}}, x_{n_{j}}\right) \rightarrow \chi\left(t^{\prime \prime}, x\right)$, so $s=\chi\left(t^{\prime}, x\right)=\chi\left(t^{\prime \prime}, x\right)$ as $s_{n} \rightarrow s$. This gives a contradiction, as $\chi(\cdot, x)$ is bijective. Let $t_{n} \rightarrow t$. Then $\chi(t, x)=s$ as $s_{n}=\chi\left(t_{n}, x_{n}\right) \rightarrow \chi(t, x)$.

To finish the proof we note that

$$
\begin{aligned}
\pi_{1}\left(s_{n}, x_{n}\right)=\pi_{1}\left(\chi\left(t_{n}, x_{n}\right), x_{n}\right)=\pi_{0}\left(t_{n}, x_{n}\right) & \rightarrow \pi_{0}(t, x) \\
& =\pi_{1}(\chi(t, x), x)=\pi_{1}(s, x) .
\end{aligned}
$$

Remark. The assumption $\chi(t, x) \geq t$ seems to be artificial. Nevertheless, it is used in the proof. It would be possible to prove the lemma without this assumption using the equality $\chi(\alpha+\beta, x)=\chi(\beta, z)+\chi\left(\alpha, \pi_{0}(\beta, x)\right)$. However, it may not be true. It is not difficult to prove this equality for a regular point $x$ (a point $x$ is regular if $\pi(\cdot, x)$ is injective). This equality 
is important in some aspects of the theory of isomorphisms of dynamical systems (see [AV, 2.4]).

COROllary 2. Let $\left(X, \pi_{0}\right)$ be a dynamical system and $\left(X, \pi_{1}\right)$ a pseudodynamical system. Let $\chi: \mathbb{R} \times X \rightarrow \mathbb{R}$ be as in the definition of an isomorphism of dynamical systems. Assume also that $\chi(t, x) \geq t$ for any $x$ and $t \geq 0$. Then $\pi_{1}$ is continuous, i.e. $\left(X, \pi_{1}\right)$ is a dynamical system, and it is isomorphic to $\left(X, \pi_{0}\right)$ as a dynamical system.

Proof. By Lemma $2,\left(X, \pi_{1}\right)$ is a semidynamical system with negative uniqueness; moreover, $N(x)=+\infty$ for each $x$. Now apply Lemma 1.

REMARK. We may define isomorphism between pseudo-semidynamical systems by replacing the condition that $\chi(\cdot, x)$ is a homeomorphism by the condition that $\chi(\cdot, x)$ is bijective and omitting the requirement of the continuity of $\chi$. Thus Lemma 2 says that if an isomorphism between pseudosemidynamical systems $\left(X, \pi_{0}\right)$ and $\left(X, \pi_{1}\right)$ satisfies these two omitted conditions and $\left(X, \pi_{0}\right)$ is a semidynamical system then so is $\left(X, \pi_{1}\right)$. Corollary 2 leads to an analogous assertion for dynamical systems.

Lemma 3. In the impulsive system $(X, \pi, M, I)$ any segment of trajectory $\pi\left(\left[t_{1}, t_{2}\right], x\right)$ contains only a finite number of points of $M$.

Proof. Suppose not. Then we can find an infinite sequence of points of $M \cap \pi\left(\left[t_{1}, t_{2}\right], x\right)$ convergent to some $\pi(s, x)$. We have $\pi(s, x) \in M$ as $M$ is closed, which contradicts the condition that points of $M$ are isolated on each trajectory.

\section{Main theorems}

TheOrem 1. Let $X$ be a metric space and $\left(X, \pi_{0}, M, I\right)$ an impulsive system given by a dynamical system (or by a semidynamical system with global backward extension) $\left(X, \pi_{0}\right)$. Assume also that:

(1) $I(M) \cap M=\emptyset$,

(2) the map I is closed,

(3) any $x \in M$ satisfies (STC).

Then the system $\left(X, \pi_{0}\right)$ is isomorphic to a dynamical (or semidynamical) system $\left(X, \pi_{1}\right)$ such that in the impulsive system $\left(X, \pi_{1}, M, I\right)$ for any $x \in X$ we have $\widetilde{\omega}_{1}(x)=+\infty$; in other words, there exists a time reparametrization of $\left(X, \pi_{0}\right)$ which gives $\left(X, \pi_{1}\right)$ with $\widetilde{\omega}_{1}(x)=+\infty$ for each $x \in X$.

Theorem 2. Let $X$ be a metric space and $\left(X, \pi_{0}, M, I\right)$ an impulsive system given by a dynamical system (or by a semidynamical system with global backward extension $)\left(X, \pi_{0}\right)$. Assume also that:

(1) $I(M) \cap M=\emptyset$, 
(2) the map I is closed,

(3) any $x \in M$ satisfies (STC).

Then there exists a topology on $X$ (the space $X$ equipped with this topology will be denoted by $\widetilde{X}$ ) such that:

(A) the new topology and the original topology on $X$ are locally identical at any $x \notin M \cup I(M)$,

(B) $\left(\widetilde{X}, \widetilde{\pi}_{0}\right)$ (where $\tilde{\pi}_{0}$ is defined by the original impulsive system) is a local semidynamical system; if $\left(X, \pi_{0}\right)$ is a dynamical system, then the set of start points of $\left(\widetilde{X}, \widetilde{\pi}_{0}\right)$ is equal to $M$ and the induced system $\left(\tilde{X} \backslash M, \tilde{\pi}_{0}\right)$ is a local semidynamical system without start points,

(C) $\tilde{\pi}_{0}=\pi_{0}$ on each segment of any trajectory of $\pi_{0}$ provided that this segment is disjoint from $M$.

Moreover, $\left(\widetilde{X}, \tilde{\pi}_{0}\right)$ has the following properties:

(a) $\widetilde{X}$ is metrizable if and only if $I^{-1}(x)$ is compact for any $x$,

(b) $\left(\widetilde{X}, \tilde{\pi}_{0}\right)$ is isomorphic to a global system $\left(\tilde{X}, \widetilde{\pi}_{1}\right)$.

The topology on $\widetilde{X}$ is obtained as a quotient topology induced by an upper semicontinuous decomposition of the space $X$ suitably modified.

We will prove both theorems together.

STEP 1. Construction of $U, U^{+}$and $U^{-}$. For any $x \in M$ take a section $S_{x}$ through $x$ and an $\varepsilon_{x} \in(0,1)$ as in the definition of section and condition (STC). Let $L_{x}$ be a bar for this section; then $S_{x}=F_{0}\left(\varepsilon_{x}, L_{x}\right)$. Define $J_{x}=$ $F_{0}\left(\varepsilon_{x} / 2, L_{x}\right)$ and consider $G_{x}=F_{0}\left(\left(0, \varepsilon_{x}\right), J_{x}\right)$. The set $G_{x}$ is an $\varepsilon_{x} / 2$-tube for the section $S_{x}$ with bar $J_{x}$ (cf. [C4]). Also $G_{x}$ is an STC-tube through $x$. We have $S_{x}=M \cap G_{x}$.

Note that if $\left(X, \pi_{0}\right)$ is a dynamical system, then we have $G_{x}=$ $\pi_{0}\left(\left(-\varepsilon_{x} / 2, \varepsilon_{x} / 2\right), S_{x}\right)$. Put

There exists an open neighbourhood $U_{x}$ of $x$ with $U_{x} \subset F_{0}\left(\left(0, \varepsilon_{x}\right), J_{x}\right)$.

$$
U_{x}^{+}=U_{x} \cap F_{0}\left(\left(0, \varepsilon_{x} / 2\right), J_{x}\right), \quad U_{x}^{-}=U_{x} \cap F_{0}\left(\left(\varepsilon_{x} / 2, \varepsilon_{x}\right), J_{x}\right) .
$$

If $\left(X, \pi_{0}\right)$ is a dynamical system, the above condition gives

$$
U_{x}^{+}=U_{x} \cap \pi_{0}\left(\left(0, \varepsilon_{x} / 2\right), S_{x}\right), \quad U_{x}^{-}=U_{x} \cap \pi_{0}\left(\left(-\varepsilon_{x} / 2,0\right), S_{x}\right) .
$$

We show that

$$
U_{x}^{+} \cap U_{y}^{-}=\emptyset \quad \text { for any } x, y \in M .
$$

Suppose to the contrary that $z \in U_{x}^{+} \cap U_{y}^{-}$. Thus there are $z_{1} \in S_{x}, z_{2} \in S_{y}$, $\alpha \in\left(0, \varepsilon_{x} / 2\right)$ and $\beta \in\left(0, \varepsilon_{y} / 2\right)$ such that $\pi_{0}\left(\alpha, z_{1}\right)=z$ and $\pi_{0}(\beta, z)=z_{2}$ (the existence of $\alpha$ and $z_{1}$ follows from the property of global backward extension of $\left.\left(X, \pi_{0}\right)\right)$. Therefore $\pi_{0}\left(\alpha+\beta, z_{1}\right)=z_{2}$ and $\alpha+\beta \in\left(0, \varepsilon_{x} / 2+\varepsilon_{y} / 2\right)$. 
If $\varepsilon_{y}<\varepsilon_{x}$ then $\alpha+\beta<\varepsilon_{x}$ and $z_{2}=\pi_{0}\left(\alpha+\beta, z_{1}\right) \in M \cap G_{x}$; however, $z_{2} \notin S_{x}$, a contradiction.

If $\varepsilon_{x}<\varepsilon_{y}$ then $\alpha+\beta<\varepsilon_{y}$ and $z_{1} \in F_{0}\left((\alpha+\beta), z_{2}\right)$, so $z_{1} \in\left(M \cap G_{y}\right) \backslash S_{y}$, which is also impossible.

Now we define

$$
\begin{aligned}
U & =\bigcup\left\{U_{x}: x \in M\right\}, \\
U^{+} & =\bigcup\left\{U_{x}^{+}: x \in M\right\}, \quad U^{-}=\bigcup\left\{U_{x}^{-}: x \in M\right\} .
\end{aligned}
$$

Then:

(1.4) $U$ is a neighbourhood of any $x \in M$,

(1.5) $U, U^{+}$and $U^{-}$are open in $X$,

(1.6) $U=U^{+} \cup U^{-} \cup M$,

(1.7) $U^{+}, U^{-}$and $M$ are pairwise disjoint,

(1.8) for any $p \in U^{-}$there are $\lambda_{1}, \lambda_{2}>0$ such that $\lambda_{1}<\lambda_{2}, \pi_{0}\left(\left[0, \lambda_{2}\right), p\right)$ $\cap U^{-}=\pi_{0}\left(\left[0, \lambda_{1}\right), p\right)$ and $\pi_{0}\left(\lambda_{1}, p\right) \in M$.

SteP 2. Construction of $\widehat{X}$. We define two new sets $M^{+}$and $M^{-}$isometric with $M$. Roughly speaking, we "double" $M$. Precisely, let $M^{+}$and $M^{-}$be disjoint sets, each disjoint from $X$, such that there exists a function $f: M^{+} \cup M^{-} \rightarrow M$ with the property that $\left.f\right|_{M^{+}}: M^{+} \rightarrow M$ is an isometry and $\left.f\right|_{M^{-}}: M^{-} \rightarrow M$ is an isometry. We will use $f$ in what follows.

Now we define $\widehat{X}=(X \backslash M) \cup M^{+} \cup M^{-}$with the topology generated by the neighbourhood system $\{\mathcal{B}(x): x \in \widehat{X}\}$, where $\mathcal{B}(x)=$

$$
\begin{cases}\{B(x, 1 / n) \backslash M: n \in \mathbb{N}\} & \text { for } x \in X \backslash M, \\ \left\{\left(B(f(x), 1 / n) \cap U^{+}\right) \cup\left(B(x, 1 / n) \cap M^{+}\right): n \in \mathbb{N}\right\} & \text { for } x \in M^{+}, \\ \left\{\left(B(f(x), 1 / n) \cap U^{-}\right) \cup\left(B(x, 1 / n) \cap M^{-}\right): n \in \mathbb{N}\right\} & \text { for } x \in M^{-},\end{cases}
$$

the balls $B(x, 1 / n)$ and $B(f(x), 1 / n)$ being considered in the space $X$.

The verification that this is a neighbourhood base (see e.g. [E, 1.2.3]) is easy. For the property: "if $x \in G \in \mathcal{B}(y)$ then there is a $V \in \mathcal{B}(x)$ with $V \subset G^{\prime \prime}$ notice that if $y \in M^{+}$and $x \in M^{+} \cup M^{-}$then $x \in M^{+}$.

In this topology, $M^{+} \cup U^{+}$is a neighbourhood of any $x \in M^{+}$, and $M^{-} \cup U^{-}$is a neighbourhood of any $x \in M^{-}$. The sets $M^{+}$and $M^{-}$are closed in $\widehat{X}$.

STEP 3. Metrizability of $\widehat{X}$. We prove that $\widehat{X}$ is metrizable by using the Nagata Theorem on metrization $([\mathrm{N}]$; see also $[\mathrm{E}, 5.4 . \mathrm{F}])$. It is enough to prove that

(3.1) $\widehat{X}$ is a $T_{0}$ space

and that for every $x \in \widehat{X}$ there exist sequences $K_{1}(x), K_{2}(x), \ldots, K_{1}^{\prime}(x)$, $K_{2}^{\prime}(x), \ldots$ of subsets of $\widehat{X}$ satisfying the following conditions: 
(3.2) $x \in \operatorname{Int} K_{n}(x) \cap \operatorname{Int} K_{n}^{\prime}(x)$,

(3.3) for any neighbourhood $G$ of $x$ there exists an $n$ such that $K_{n}(x) \subset G$,

(3.4) $\quad K_{n}^{\prime}(x) \cap K_{n}^{\prime}(y)=\emptyset$ whenever $y \notin K_{n}(x)$,

(3.5) $\quad K_{n}^{\prime}(y) \subset K_{n}(x)$ whenever $y \in K_{n}(x)$.

The condition (3.1) is trivial, as $\widehat{X}$ is obviously a Hausdorff space. Now we define the families $\left\{K_{n}(x): n \in \mathbb{N}\right\}$ and $\left\{K_{n}^{\prime}(x): n \in \mathbb{N}\right\}$. First, let $x \notin M^{+} \cup M^{-}$. For $x \notin U$ put

$$
r_{x}=\min \{n: B(x, 1 / n) \cap M=\emptyset\}, \quad \varrho_{x}=\min \left\{1 / 2 n, r_{x}\right\},
$$

for $x \in U$ put

$$
\begin{array}{lll}
r_{x}=\min \left\{n: B(x, 1 / n) \subset U^{+} \backslash M\right\}, & \varrho_{x}=\min \left\{1 / 2 n, r_{x}\right\} & \text { if } x \in U^{+}, \\
r_{x}=\min \left\{n: B(x, 1 / n) \subset U^{-} \backslash M\right\}, & \varrho_{x}=\min \left\{1 / 2 n, r_{x}\right\} & \text { if } x \in U^{-},
\end{array}
$$

and for all $x \notin M^{+} \cup M^{-}$set

$$
\begin{aligned}
& K_{n}(x)=(B(x, 1 / n) \backslash M) \cup f^{-1}(B(x, 1 / n) \cap M), \\
& K_{n}^{\prime}(x)=B\left(x, \varrho_{x}\right) .
\end{aligned}
$$

Now let $x \in M^{+} \cup M^{-}$. Then define $r_{x}=\min \{n: B(f(x), 1 / n) \subset U\}$. For $x \in M^{+}$denote by $x^{-}$the unique element of $M^{-}$such that $f\left(x^{-}\right)=f(x)$. If $n \leq r_{x}$ put

$$
\begin{aligned}
& K_{n}(x)=(B(f(x), 1 / n) \backslash M) \cup\left(B(x, 1 / n) \cap M^{+}\right) \cup\left(B\left(x^{-}, 1 / n\right) \cap M^{-}\right), \\
& K_{n}^{\prime}(x)=\left(B(f(x), 1 / 2 n) \backslash\left(M \cup U^{-}\right)\right) \cup\left(B(x, 1 / 2 n) \cap M^{+}\right),
\end{aligned}
$$

and if $n>r_{x}$ put

$$
\begin{aligned}
& K_{n}(x)=\left(B(f(x), 1 / n) \cap U^{+}\right) \cup\left(B(x, 1 / n) \cap M^{+}\right), \\
& K_{n}^{\prime}(x)=\left(B(f(x), 1 / 2 n) \cap U^{+}\right) \cup\left(B(x, 1 / 2 n) \cap M^{+}\right) .
\end{aligned}
$$

For $x \in M^{-}$we define $K_{n}(x)$ and $K_{n}^{\prime}(x)$ analogously.

Now, the conditions (3.2) and (3.3) are obvious. The verification of (3.4) and (3.5) is not difficult but technical and requires the analysis of many cases, and is therefore omitted.

STEP 4. Construction of $\tilde{X}$. We now define an equivalence relation $\mathcal{R}$ on $\widehat{X}$ by describing its equivalence classes $[x]$ for $x \in \widehat{X}$.

Recall that $M \cap I(M)=\emptyset$. If $x \notin M^{-} \cup I(M)$ then we set $[x]:=\{x\}$. If $x \in I(M)$ then $[x]:=\{x\} \cup\left(M^{-} \cap f^{-1}\left(I^{-1}(x)\right)\right)$. Finally, if $x \in M^{-}$then $[x]:=[I(f(x))]$. The idea is to glue any point of $I(M)$ with all the points from $M^{-}$which "jump" to that point. The set $I^{-1}(x)$ is closed in $M$ as $I$ is continuous. Therefore $M^{-} \cap f^{-1}\left(I^{-1}(x)\right)$ is closed in $M^{-}$, so it is closed in $\widehat{X}$. Thus the equivalence class of any point is closed, as $\widehat{X}$ is a $T_{1}$ space.

Now consider the space $\widehat{X} / \mathcal{R}$ with the quotient topology. We have a natural bijection between $X$ and $\widehat{X} / \mathcal{R}$. Indeed, we can identify the points 
of $M$ with the one-point classes given by suitable points of $M^{+}$, and the points of $I(M)$ with the classes given by these points. Thus we can consider $\widehat{X} / \mathcal{R}$ with the quotient topology as $X$ with a new topology, which will be denoted by $\widetilde{X}$. In fact, we show that the decomposition of $\widehat{X}$ corresponding to $\mathcal{R}$ is upper semicontinuous (see [CV], [E]).

STEP 5. Closedness of the natural map $p: \widehat{X} \rightarrow \widehat{X} / \mathcal{R}$. First we prove

Lemma. Any subset $F$ of $X$ disjoint from $M$ is closed in $X$ if and only if it is closed in $\widehat{X}$.

Assume that $F$ is closed in $X$. We need to show that for any $y \notin F$ there exists a $V \in \mathcal{B}(y)$ with $V \cap F=\emptyset$. Suppose $y \notin M^{+} \cup M^{-}$. There is an $n$ such that $B(y, 1 / n) \cap F=\emptyset$, so we can set $V:=B(y, 1 / n) \backslash M$. If $y \in M^{+} \cup M^{-}$, then there exists an $n$ such that $B(f(y), 1 / n) \cap F=\emptyset$ and we set $V:=B(f(y), 1 / n) \cap U^{ \pm}$for $y \in M^{ \pm}$.

Conversely, let $F$ be closed in $\widehat{X}$. We need to show that for any $y \notin F$ there exists an $n$ such that $B(y, 1 / n) \cap F=\emptyset$. Assume that $y \notin M$. For $k$ large enough we have $B(x, 1 / k) \backslash M=B(x, 1 / k)$ as $M$ is closed. Thus for some large $k$ we have $B(y, 1 / k) \cap F=\emptyset$. Now fix $y \in M$. Let $y^{+} \in M^{+}$ and $y^{-} \in M^{-}$be such that $f\left(y^{+}\right)=y$ and $f\left(y^{-}\right)=y$. We can find a $k$ such that $\left(B\left(f\left(y^{+}\right), 1 / k\right) \cap U^{+}\right) \cap F=\emptyset,\left(B\left(f\left(y^{-}\right), 1 / k\right) \cap U^{-}\right) \cap F=\emptyset$ and $(B(y, 1 / k) \cap M) \cap F=\emptyset$. Therefore $(B(y, 1 / k) \cap U) \cap F=\emptyset$. For $n$ sufficiently large we have $B(y, 1 / n) \cap F=\emptyset$ as $U$ is open in $X$. We have thus proved the lemma.

From the lemma it follows that $I(M)$ is closed in $\widehat{X}$ as $I$ is closed and $M \cap I(M)=\emptyset$. Thus $I(M) \cup M^{-}$is closed in $\widehat{X}$.

Now we show that the restriction of the natural quotient map $p: \widehat{X} \rightarrow$ $\widehat{X} / \mathcal{R}$ to $I(M) \cup M^{-}$is closed. Take a closed set $A \subset I(M) \cup M^{-}$. Then $A$ is the disjoint union $A^{-} \cup A_{I}$, where $A^{-}=M^{-} \cap A$ and $A_{I}=I(M) \cap A$. Since $A_{I}$ is closed in $I(M)$, it is closed in $\widehat{X}$, and analogously $A^{-}$is closed in $\widehat{X}$. We have $p\left(A^{-}\right)=I\left(f\left(A^{-}\right)\right) \cup\left(f^{-1}\left(I^{-1}\left(I\left(f\left(A^{-}\right)\right)\right)\right) \cap M^{-}\right)$. The first summand is closed in $X$ and disjoint from $M$, so closed in $\widehat{X}$; the second is closed in $M^{-}$, so also in $\widehat{X}$. Thus $p\left(A^{-}\right)$is closed in $\widehat{X}$. Moreover, $p\left(A_{I}\right)=$ $A_{I} \cup\left(f^{-1}\left(I^{-1}\left(A_{I}\right)\right) \cap M^{-}\right)$is again closed in $\widehat{X}$. Therefore $p(A)$ is closed and the decomposition of $I(M) \cup M^{-}$is upper semicontinuous (see [E, 2.4] or $[\mathrm{CV}, 18 . \mathrm{A} .12])$.

It follows that $p: \widehat{X} \rightarrow \widehat{X} / \mathcal{R}$ is closed. Indeed, by [E, 2.4.13] it is enough to find a closed subspace $\widehat{Z}$ of $\widehat{X}$ such that $\mathcal{R}$ gives a decomposition of $\widehat{X}$ into one-point sets $\{z\}$ with $z \in \widehat{X} \backslash \widehat{Z}$ and such that the quotient mapping restricted to $\widehat{Z}$ is closed. We take $\widehat{Z}=I(M) \cup M^{-}$. We have shown that the decomposition of $\widehat{X}$ corresponding to $\mathcal{R}$ is upper semicontinuous. 
STEP 6 . Normality of $\widetilde{X} \times[0,1]$. First notice that $\widetilde{X}$ is perfectly normal, as it is metrizable (see for instance [E, 4.1.13]). Perfect normality is invariant under continuous closed mappings $([\mathrm{E}, 1.5 .20])$, so $\widehat{X} / \mathcal{R}$ is also perfectly normal. Using $[\mathrm{E}, 5.2 .5]$ we conclude that $\widehat{X} / \mathcal{R}$ is countably paracompact and according to $[\mathrm{E}, 5.2 .8]$ the space $\widetilde{X} \times[0,1]$ is normal.

Note that $\widetilde{X}$ need not be metrizable. According to the Hanai-MoritaStone Theorem ([E, 4.4.17]) applied to $p$ the space $\widehat{X} / \mathcal{R}$ is metrizable if and only if the boundary of $p^{-1}(y)$ is compact for any $y \in \widehat{X} / \mathcal{R}$.

We show that the last property holds if and only if $I^{-1}(x)$ is compact for any $x$.

It is enough to consider the case where $x \in \widehat{X} / \mathcal{R}$ is the equivalence class of a point $y \in I(M)$. First note that the closed set $M$ has empty interior. Therefore for any $x$ the boundary of $I^{-1}(x)$ (which is closed) is equal to $I^{-1}(x)$.

The set $p^{-1}(x)$ is the union of a one-point-set $\{y\}(y \in I(M))$ and a subset $I_{y}$ of $M^{-}$. The point $y$ is separated from the closed set $M$, so $\operatorname{Fr}\left(\{y\} \cup I_{y}\right)=\{y\} \cup I_{y}$.

Assume that $I^{-1}(y)$ is compact. Then $f^{-1}\left(I^{-1}(y)\right)=I_{y}$ is compact in $M^{-}$, so in $\widehat{X}$. Thus $\operatorname{Fr} p^{-1}(x)=I_{y} \cup\{y\}$ is compact in $\widehat{X}$.

Now let $\operatorname{Fr} p^{-1}(x)=I_{y} \cup\{y\}$ be compact in $\widehat{X}$. Using the lemma from Step 5 we get the compactness of $I_{y}$ and hence of $I^{-1}(y)$.

STEP 7. Construction of the semidynamical system $\left(\widetilde{X}, \widetilde{\pi}_{0}\right)$. The semitrajectory $\widetilde{\pi}_{0}$ of a point $x \in \widetilde{X}$ is defined for any $t \in\left[0, \widetilde{\omega}_{0}\left(q_{x}\right)\right)$ according to the construction of $\widetilde{\pi}_{0}$. Note that any $x \in M$ is a start point of this system.

The initial value axiom and the semigroup axiom for $\widetilde{\pi}_{0}$ are obvious. We need to show

(7.1) Kamke's axiom, i.e. the openness of $\operatorname{dom}_{\widetilde{\pi}_{0}}$ in $\mathbb{R}_{+} \times \tilde{X}$,

(7.2) the continuity of $\widetilde{\pi}_{0}$.

For the proof of (7.1) and (7.2), we need some preparation.

For any $y \in U^{-}$there exist unique $p_{y} \in M$ and unique $\mu_{y}$ such that $p_{y}=$ $\pi_{0}\left(\mu_{y}, y\right)$ and $\pi_{0}\left(\left(0, \mu_{y}\right), y\right) \cap M=\emptyset$. Thus we can define $g: U^{-} \cup M^{-} \rightarrow M$ and $T: U^{-} \cup M^{-} \rightarrow \mathbb{R}$ by

$$
g(y)=\left\{\begin{array}{ll}
p_{y} & \text { for } y \in U^{-}, \\
f(y) & \text { for } y \in M^{-},
\end{array} \quad T(y)=\mu_{y} .\right.
$$

It is easy to verify that $g$ and $T$ are continuous. Therefore $I \circ g: U^{-} \cup M^{-} \rightarrow$ $X \backslash M$ is continuous. For $x \in U^{-} \cup M^{-}$set $h([x])=I(g(x))$. The function $h:\left(U^{-} \cup M^{-}\right) / \mathcal{R} \rightarrow X \backslash M$ is well defined and continuous as $I \circ g=h \circ p$ (see $[\mathrm{E}, 2.4 .2]$ ). 
In the proof of (7.1) and (7.2) the points from $\widetilde{X}$ will be considered as elements of $\widehat{X} / \mathcal{R}$.

SteP 8. Proof of Kamke's axiom for $\left(\widetilde{X}, \widetilde{\pi}_{0}\right)$. According to [P1, Lemma 1.3.1, Remark 1.3.4] the openness of $\operatorname{dom}_{\widetilde{\pi}_{0}}$ is equivalent to the lower semicontinuity of the escape time function $\omega^{*}: \widetilde{X} \ni x \mapsto \omega^{*}(x) \in(0,+\infty]$. For any $x$, we have $\omega^{*}(x)=\widetilde{\omega}_{0}(x)$, but we have different topologies in the domains of these functions.

Let first $x \in X \backslash(M \cup I(M))$. The topologies in $X$ and $\widetilde{X}$ are locally identical at $x$, so using $[\mathrm{C} 2,4.4]$ we conclude that $\omega^{*}$ is lower semicontinuous at $x$.

Now let $x \in I(M)$. Assume that $\omega^{*}([x])>r$. Take $x_{M} \in I^{-1}(x) \cap M^{-}$. There exists an open neighbourhood $V$ of $x$ which can be represented as the union $V=V_{x} \cup V^{-}$, where $V_{x}$ is a neighbourhood of $x$ in $X$ (disjoint from $M)$ and $V^{-}$is an open subset of $U^{-} \cup M^{-}$containing $f^{-1}\left(I^{-1}(x)\right) \cap M^{-}$. Any neighbourhood contained in $V$ can also be represented in that way. There exists a neighbourhood $W_{x}$ of $x$ (contained in $V$ ) such that $\omega^{*}(z)>r$ for any $z \in W_{x}$, again by Theorem 4.4 of [C2]. We can find an open subset $\widehat{W}$ of $\left(U^{-} \cup M^{-}\right) / \mathcal{R}$ containing $\left[x_{M}\right]$ such that $h(\widehat{W}) \subset W_{x}$. If $[z] \in \widehat{W}$, then $\omega^{*}([z]) \geq \omega^{*}([g(z)])=\widetilde{\omega}_{0}(I(g(z)))>r\left(\right.$ we have $\left.I(g(z))=h([g(z)]) \in W_{x}\right)$. Take a neighbourhood $W=W_{x} \cup \widehat{W}$ of $x$; then $\omega^{*}(y)>r$ for any $y \in W$. We have thus shown the lower semicontinuity of $\omega^{*}$ at $x$.

We are left with the case where $x \in M^{+}$. There is a neighbourhood of $[x]$ consisting only of one-point equivalence classes given by points of $U^{+} \cup M^{+}$. Denote the subset of $X$ consisting of those points by $V$. Now the set $D=\left(U_{f(x)} \backslash U_{f(x)}^{-}\right) \cap V$ is a neighbourhood of $f(x)$ in $U^{+} \cup M$ (see Step 1). Thus $\widetilde{D}=\left(f^{-1}(M \cap D) \cap M^{+}\right) \cup\left(U_{f(x)}^{+} \cap D\right)$ is a neighbourhood of $[x]$ in $\widetilde{X}$.

Assume now again that $\omega^{*}([x])=\widetilde{\omega}_{0}(f(x))>r$. There is a $\delta$ such that $\pi_{0}(\delta, f(x)) \in D, \pi_{0}((0, \delta), f(x)) \subset U^{+}$and $\widetilde{\omega}_{0}\left(\pi_{0}(\delta, f(x))\right)>r$. Using again $\left[\right.$ C.2, 4.4] we can find a neighbourhood $W$ of $\pi_{0}(\delta, f(x))$ with $W \subset D$ and $\widetilde{\omega}_{0}(z)>r$ for any $z \in W$. Then for any $[z] \in\left(F_{0}(\delta, W) \cap U^{+}\right) \cup\left(f^{-1}(M \cap\right.$ $\left.\left.F_{0}(\delta, W)\right) \cap M^{+}\right)$we have $\omega^{*}([z])>r+\delta>r$.

\section{STEP 9. Continuity of $\widetilde{\pi}_{0}$}

9.1. First take an $x \in X \backslash(M \cup I(M))$. The topology in $\widetilde{X}$ is locally identical at $[x]$ with the metric topology on $X$, so we may use sequences to check continuity. Let $t \geq 0$. By Lemma $3, \widetilde{\pi}_{0}([0, t), x)$ contains only a finite number of impulse points. If there is none, the continuity at $(t,[x])$ is obvious. Assume that there is one impulse point with $\phi(x)=s$; we have $\pi_{0}(s, x) \in M, \pi_{0}([0, s), x) \cap M=\emptyset$. Take sequences $t_{n} \rightarrow t$ and $\left[x_{n}\right] \rightarrow[x]$; for large $n,\left[x_{n}\right]$ are one-point sets and $x_{n} \rightarrow x$.

We show that there exists a sequence $\left(\delta_{n}\right)$ converging to 0 such that $0<s+\delta_{n}<t_{n}, \pi_{0}\left(s+\delta_{n}, x_{n}\right) \in M$ and $\pi_{0}\left(\left(0, s+\delta_{n}\right), x_{n}\right) \cap M=\emptyset$. 
There is a $\varrho$ such that $z=\pi_{0}(s-\varrho, x) \in U^{-}$and $\pi_{0}([0, s-\varrho), x) \cap M=\emptyset$. Thus we can find an open set $W$ containing $\pi_{0}([0, s-\varrho), x)$ and disjoint from $M$. Therefore for $n$ large enough we have $\pi_{0}\left([0, s-\varrho), x_{n}\right) \cap M=\emptyset$ and $z_{n}=\pi_{0}\left(s-\varrho, x_{n}\right) \subset U^{-}$. There exists a sequence $\left(\varrho_{n}\right)$ with $\pi_{0}\left(\varrho_{n}, z_{n}\right) \in M$ and $\pi_{0}\left(\left[0, \varrho_{n}\right), z_{n}\right) \cap M=\emptyset$ for $n$ sufficiently large. It is easy to prove that $\varrho_{n} \rightarrow \varrho$ as $\pi_{0}(\varrho, z) \in M$ and $\pi_{0}([0, \varrho), z) \cap M=\emptyset\left(\left(\varrho_{n}\right)\right.$ is bounded and cannot have a subsequence convergent to a number different from $\varrho)$. The sequence $\delta_{n}=\varrho_{n}-\varrho$ is as required.

Now we conclude that $y_{n}=\pi_{0}\left(s+\delta_{n}, x_{n}\right) \rightarrow \pi_{0}(s, x)$. By the continuity of $I$ we have $y_{n}^{+} \rightarrow \pi_{0}(s, x)^{+}$and $\widetilde{\pi}_{0}\left(t_{n}, x_{n}\right)=\pi_{0}\left(t_{n}-s-\delta_{n}, y_{n}^{+}\right) \rightarrow \pi_{0}(t-s$, $\left.\pi_{0}(s, x)^{+}\right)=\widetilde{\pi}_{0}(t, x)$, so $\left[\widetilde{\pi}_{0}\left(t_{n}, x_{n}\right)\right] \rightarrow\left[\widetilde{\pi}_{0}(t, x)\right]$.

When there are more impulse points on $\widetilde{\pi}([0, t), x)$ we apply the above procedure finitely many times.

9.2. For $[x] \in M^{+} / \mathcal{R}$ the proof goes in the same manner.

9.3. Now take $[x]$ for $x \in I(M)$ and $t>0$ such that $\pi_{0}([0, t], x) \cap M=\emptyset$. Any neighbourhood $\widetilde{G}$ of $\pi_{0}(t, x)$ contains a subset $\widetilde{G}_{0}$ consisting only of onepoint equivalence classes such that $G_{0}=\left\{y:[y] \in \widetilde{G}_{0}\right\}$ is a neighbourhood of $\pi_{0}(t, x)$ in $X$.

There exists a neighbourhood $V_{0}$ of $x$ and a $\delta>0$ such that $\pi_{0}((t-\delta$, $\left.t+\delta), V_{0}\right) \subset G_{0}$. As in Step 8, we can find a neighbourhood $\widehat{W}_{0}$ of $[x]$ in $\left(U^{-} \cup M^{-}\right) / \mathcal{R}$ such that $I(g(z)) \in G_{0}$ for any $[z] \in \widehat{W}_{0}$. Define $W_{1}=\{z \in$ $\left.U^{-} \cup M^{-}: T(z)<\delta / 2\right\}=T^{-1}(-\infty, \delta / 2)$. Then $W_{1}$ is open in $U^{-} \cup M^{-}$ and $M^{-} \subset W_{1}$. Thus $\widetilde{W}_{1}=W_{1} / \mathcal{R}$ is a neighbourhood of $M^{-} / \mathcal{R}$ in $\left(U^{-} \cup\right.$ $\left.M^{-}\right) / \mathcal{R}$. Now take $\widetilde{V}=\widetilde{V}_{0} \cap \widetilde{W}_{0} \cap \widetilde{W}_{1}$. For any $[z] \in \widetilde{V}$ and $s \in(t-\delta / 2, t+\delta / 2)$ we have $\widetilde{\pi}_{0}(s,[z]) \in \widetilde{G}_{0}$. Indeed, for $z \in V_{0}$ this is obvious. Now take $[z] \in$ $\widetilde{W}_{0} \cap \widetilde{W}_{1}$. We can find a $u \in[0, \delta / 2)$ with $\pi_{0}(u, z) \in M$, so $\left(\pi_{0}(u, z)\right)^{+} \in V_{0}$. Now $\widetilde{\pi}_{0}(s,[z])=\widetilde{\pi}_{0}\left(s-u,\left[\left(\widetilde{\pi}_{0}(u, z)\right)^{+}\right]\right) \in \widetilde{G}_{0}$ as $s-u \in(t-\delta, t+\delta / 2)$.

9.4. For any $x$ and $t<\omega^{*}(x)$, the continuity of $\widetilde{\pi}_{0}$ at $(t, x)$ follows immediately by applying 9.1-9.3.

We have proved assertions (A)-(C) of Theorem 2 as well as assertion (a). Assertion (b) is a consequence of the next step.

STEP 10. The system $\left(\widetilde{X}, \widetilde{\pi}_{1}\right)$ and an isomorphism between $\left(\widetilde{X}, \widetilde{\pi}_{1}\right)$ and $\left(\widetilde{X}, \widetilde{\pi}_{0}\right)$. Since $[0,1] \times \widetilde{X}$ is normal (see Step 6$)$, we may apply the results of [Ca]. It follows that there exist a global semidynamical system $\left(\widetilde{X}, \widetilde{\pi}_{1}\right)$ and a time reparametrization $\widetilde{\chi}$ from $\left(\widetilde{X}, \widetilde{\pi}_{0}\right)$ to $\left(\widetilde{X}, \widetilde{\pi}_{1}\right)$. Precisely, by Theorem 0 , there is a function $\tilde{\chi}: \operatorname{dom}_{\widetilde{\pi}_{0}} \rightarrow[0,+\infty)$ satisfying the following conditions:

$$
\begin{aligned}
& \tilde{\chi}(0, x)=0 \text { for any } x, \\
& \widetilde{\chi}(\cdot, x):[0, \widetilde{\omega}(x)) \rightarrow[0,+\infty) \text { is a homeomorphism for any } x,
\end{aligned}
$$




$$
\begin{array}{ll}
(10.3) & \widetilde{\pi}_{0}(t, x)=\widetilde{\pi}_{1}(\widetilde{\chi}(t, x), x) \text { for any }(t, x) \in \operatorname{dom}_{\widetilde{\pi}_{0}}, \\
(10.4) & \widetilde{\chi}(t, x) \geq t \text { for any }(t, x) \in \operatorname{dom}_{\tilde{\pi}_{0}} .
\end{array}
$$

The proof of Theorem 2 is now finished.

STEP 11. Final approach to the assertion of Theorem 1. We will show the existence of a semidynamical system $\left(X, \pi_{1}\right)$ and a function $\chi: \mathbb{R}_{+} \times X \rightarrow$ $\mathbb{R}_{+}$satisfying the following conditions:

(11.1) $\chi$ is continuous,

(11.2) $\quad \chi(0, x)=x$ for any $x$,

$(11.3) \quad \chi(\cdot, x):[0,+\infty) \rightarrow[0,+\infty)$ is a homeomorphism for any $x$,

(11.4) $\quad \pi_{0}(t, x)=\pi_{1}(\chi(t, x), x)$ for any $t \geq 0, x \in X$,

(11.5) for any $x \in X$ the escape time $\widetilde{\omega}_{1}(x)$ in the impulsive system $\left(X, \pi_{1}, M, I\right)$ is equal to $+\infty$.

This will finish the proof of Theorem 1 in the case where $\left(X, \pi_{0}\right)$ is a semidynamical system with global backward extension.

If $\left(X, \pi_{0}\right)$ is a dynamical system, then to get the assertion of Theorem 1 we need to show that $\left(X, \pi_{1}\right)$ is also dynamical. To prove this we show that two additional conditions are also satisfied:

(11.6) if $\left(X, \pi_{0}\right)$ has negative uniqueness then so does $\left(X, \pi_{1}\right)$,

(11.7) for any $x \in X$ the negative escape time of $x$ in $\pi_{1}$ is $+\infty$.

Then applying Lemma 1 we will get the required dynamical system and the required isomorphism, which will finish the proof of Theorem 1.

SteP 12. Construction of $\chi$ and $\pi_{1}$. Fix $x \in X$. Using Lemma 3 we deduce that

(12.1) for any $\alpha \geq 0$ there is a $\delta$ such that

$$
\left.\pi_{0}((\alpha-\delta, \alpha), x) \cup \pi_{0}(\alpha, \alpha+\delta), x\right) \cap M=\emptyset .
$$

Thus we may proceed with the following construction. Suppose $M^{\oplus}(x)$ $\neq \emptyset$. Then we set $x^{\star 0}=x$, we have $\pi_{0}\left(\left(0, \phi\left(x^{\star 0}\right)\right), x^{\star 0}\right) \cap M=\emptyset$ and $x^{\star 1}=$ $x^{1}=\pi_{0}\left(\phi\left(x^{\star 0}\right), x^{\star 0}\right) \in M$ (we use the notation of the Preliminaries). Now put $x^{\star 2}=\pi_{0}\left(\phi\left(x^{\star 1}\right), x^{\star 1}\right) \in M$ and so on. Define $u^{n}=\phi\left(x^{\star 0}\right)+\cdots+$ $\phi\left(x^{\star(n-1)}\right)$; we have $\pi_{0}\left(u^{n}, x\right)=x^{\star n}$. The sequence $\left(\phi\left(x^{\star n}\right)\right)$ is either finite or infinite; in the latter case $u^{n} \rightarrow+\infty$ (if not, $\left(\pi_{0}\left(u^{n}, x\right)\right)$ converges to some element of $M$, which contradicts (12.1)) and $\sum_{n=1}^{\infty} \phi\left(x^{\star n}\right)=+\infty$.

We set $s^{k}=\tilde{\chi}\left(\phi\left(x^{\star k}\right), x^{\star k}\right)$ (if $x^{\star k}$ is the equivalence class of an element of $I(M)$ we consider it as an element of $I(M)$; this convention will also be used below).

Now we define $\chi(t, x)$ :

(12.2) If $t<\phi\left(x^{\star 0}\right)$ (in particular, if $\phi(x)=+\infty$ ) then $\chi(t, x)=\widetilde{\chi}(t, x)$. 
(12.3) If $\phi\left(x^{\star 0}\right)+\cdots+\phi\left(x^{\star(k-1)}\right) \leq t$ and either $t<\phi\left(x^{\star 0}\right)+\cdots+\phi\left(x^{\star k}\right)$ or the sequence $\left(\phi\left(x^{\star n}\right)\right)$ is finite with last term $\phi\left(x^{\star(k-1)}\right)$, then $\chi(t, x)=s^{0}+\cdots+s^{k-1}+\widetilde{\chi}\left(t-\left(\phi\left(x^{\star 0}\right)+\cdots+\phi\left(x^{\star(k-1)}\right)\right), x^{\star k}\right)$.

From the definition of $\chi,(10.2)$ and (10.4) it follows immediately that $\chi(\cdot, x)=\chi_{x}: \mathbb{R}_{+} \rightarrow \mathbb{R}_{+}$is an increasing bijection and

$$
\chi(t, x) \geq t .
$$

Now we define $\pi_{1}$ by

$$
\pi_{1}(t, x)=\pi_{0}\left(\chi_{x}^{-1}(t), x\right) .
$$

Conditions (11.2), (11.4) and (11.6) follow immediately from the definitions of $\chi$ and $\pi_{1}$. Since $\widetilde{\chi}(\cdot, x)$ is increasing for any $x$, so is $\chi(\cdot, x)$.

To prove (11.7) fix $x$ and $t>0$; we show that there is a $z$ such that $\pi_{1}(t, z)=x$. There is a $y \in X$ with $\pi_{0}(t, y)=x$, so $\pi_{1}(\chi(t, y), y)=x$. However, $\chi(t, y) \geq t$ so we can find a $z \in \pi_{1}^{+}(y)$ with $\pi_{1}(t, z)=x$.

The "impulsive motion" $\widetilde{\pi}_{1}$ given by $\pi_{1}, M$ and $I$ coincides with the motion in the semidynamical system $\left(X, \widetilde{\pi}_{1}\right)$. Hence using the above definitions and the properties of $\widetilde{\pi}_{1}$ we immediately conclude that the local existence axiom, the initial value axiom and the semigroup axiom are satisfied for $\pi_{1}$. Moreover, $\pi_{1}(t, x)$ is defined for any $t \geq 0$ and $x$, and so also is $\widetilde{\pi}_{1}(t, x)$, which gives (11.5). Thus to prove that $\left(X, \pi_{1}\right)$ is a semidynamical system we need, according to Lemma 2 , only the continuity of $\chi$.

Once we prove it, then, on account of the properties of $\chi$, also (11.3) follows.

STEP 13. Continuity of $\chi$. We have to show that if $x_{n} \rightarrow x$ in $X$ and $t_{n} \rightarrow t$ then $\chi\left(t_{n}, x_{n}\right) \rightarrow \chi(t, x)$. It is enough to consider four cases:

(i) $x \notin M$ and $x_{n} \notin M$ for any $n$,

(ii) $x \in M$ and $x_{n} \in M$ for any $n$,

(iii) $x \in M$ and $x_{n} \in U^{+}$for any $n$,

(iv) $x \in M$ and $x_{n} \in U^{-}$for any $n$.

First we prove the assertion when $t<\phi(x)=\phi\left(x^{\star 0}\right)$ or $\phi(x)=+\infty$. This will be done in 13.1-13.4.

13.1. Let (i) hold. Then, according to [C2, Ths. 2.11(1) and 3.8(1)], $\phi$ is continuous at $x$, so $\phi\left(x_{n}\right) \rightarrow \phi(x)$ (we use the property that a semidynamical system with global backward extension has no start points). Thus for large $n$ we have $t<\phi\left(x_{n}\right)$ and $t_{n}<\phi\left(x_{n}\right)$. Therefore $\chi(t, x)=\widetilde{\chi}(t, x)$ and $\chi\left(t_{n}, x_{n}\right)=\tilde{\chi}\left(t_{n}, x_{n}\right)$, so the result follows from the continuity of $\widetilde{\chi}$.

13.2. Let (ii) hold. Using [C2, Ths. 2.11(3) and 3.8(3)] and [K1, Th. 4] (cf. also [C2, Sec. 2]) we conclude that $\phi\left(x_{n}\right) \rightarrow \phi(x)$. Now the proof goes as in 13.1 . 
13.3. Consider case (iii) and take $\left(x_{n}\right)$ with $x_{n} \in U^{+}, x_{n} \rightarrow x \in M$. We can find a $\delta<\varepsilon_{x} / 2$ (where $\varepsilon_{x}$ was defined in Step 1 ) such that $\pi_{0}((0, \delta], x)$ $\subset U^{+}$. Thus $\pi_{0}\left(\delta, x_{n}\right) \in U^{+}$for large $n$. From the continuity of $\pi_{0}$, the properties of sections and the construction of $U$ we conclude that $\pi_{0}\left([0, \delta], x_{n}\right) \subset$ $U^{+}$for sufficiently large $n$. Thus $\pi_{0}\left(\delta, x_{n}\right) \rightarrow \pi_{0}(\delta, x), \phi\left(x_{n}\right)=\delta+\phi\left(\pi_{0}\left(\delta, x_{n}\right)\right)$ and $\phi(x)=\delta+\phi\left(\pi_{0}(\delta, x)\right)$. Just as in 13.1, we have $\phi\left(\pi_{0}\left(\delta, x_{n}\right)\right) \rightarrow \phi\left(\pi_{0}(\delta, x)\right)$ and from the above reasoning $\phi\left(x_{n}\right) \rightarrow \phi(x)$. Now the proof continues as in 13.1 .

13.4. Assume (iv). Take $\phi\left(x_{n}\right)$ and put $\bar{x}_{n}=\pi_{0}\left(\phi\left(x_{n}\right), x_{n}\right) \in M$. Then $\bar{x}_{n} \rightarrow x$ and $\phi\left(x_{n}\right) \rightarrow 0$ (note that $\bar{x}_{n}=x_{n}^{1}=x_{n}^{\star 1}$ ). The problem reduces to two cases: $t_{n}<\phi\left(x_{n}\right)$ for any $n$, and $t_{n} \geq \phi\left(x_{n}\right)$ for any $n$. In the first case we have $t_{n} \rightarrow 0, t=0$ and $\chi\left(t_{n}, x_{n}\right)=\tilde{\chi}\left(t_{n}, x_{n}\right) \rightarrow 0=\chi(0, x)$. Now let $t_{n} \geq \phi\left(x_{n}\right)$ for any $n$. Then $\chi\left(t_{n}, x_{n}\right)=\tilde{\chi}\left(\phi\left(x_{n}\right), x_{n}\right)+\tilde{\chi}\left(t_{n}-\phi\left(x_{n}\right), \bar{x}_{n}\right) \rightarrow$ $0+\tilde{\chi}(t, x)=\chi(t, x)$.

Now assume that $\phi(x) \leq t<\phi(x)+\phi\left(x^{\star 1}\right)$.

13.5. Let (i) hold. We need to consider two cases: $t_{n} \leq \phi\left(x_{n}\right)$ for any $n$, and $t_{n}>\phi\left(x_{n}\right)$ for any $n$. In the first case the proof goes as in 13.1. In the second case take $z_{n}=\pi_{0}\left(\psi\left(x_{n}\right), x_{n}\right)$ (we have $z_{n}=x_{n}^{1}=x_{n}^{\star 1}$ ). Then $z_{n} \rightarrow z=\pi_{0}(\psi(x), x)$. By the same reasoning as in 13.1 we can assume that $0<t_{n}-\phi\left(x_{n}\right)<\phi\left(z_{n}\right)$ for large $n$. We have $\phi\left(x_{n}\right) \rightarrow \phi(x)$. Thus $\chi\left(t_{n}, x_{n}\right)=\widetilde{\chi}\left(\phi\left(x_{n}\right), x_{n}\right)+\widetilde{\chi}\left(t_{n}-\phi\left(x_{n}\right), z_{n}\right) \rightarrow \widetilde{\chi}(\phi(x), x)+\widetilde{\chi}(t-\phi(x), z)$. On the other hand, $\chi(t, x)=\widetilde{\chi}(\phi(x), x)+\widetilde{\chi}(t-\phi(x), z)$.

13.6. In case (ii) the proof combines the reasoning from 13.2 and 13.5 , in case (iii) it combines the reasoning from 13.3 and 13.5, and in case (iv) the reasoning from 13.4 and 13.5. We have thus shown that $\chi$ is continuous at $(t, x)$, where $t<\phi(x)+\phi\left(x^{\star 1}\right)$.

Now let $t$ be arbitrary. We are left with the task of proving the assertion if $\phi\left(x^{\star 0}\right)+\cdots+\phi\left(x^{\star(k-1)}\right) \leq t$ and either $t<\phi\left(x^{\star 0}\right)+\cdots+\phi\left(x^{\star k}\right)$ or the sequence $\left(\phi\left(x^{\star n}\right)\right)$ is finite with last term $\phi\left(x^{\star(k-1)}\right)$. It is enough to apply the procedure from 13.5 and 13.6 finitely many times. Thus we have finished the proof.

Remark. Assumption (1) where we require from a point to "jump" to a point not belonging to the impulse set is natural; jumping to the impulse set would be artificial. Assumption (3) on "good position" of $M$ is important in the theory and several results are based on it (see [K1], [C2]). Only assumption (2) may at first seem to be of another kind. However, in many natural situations the function $I$ is even supposed to be a homeomorphism onto its image. 
REMARK. In some cases, the space $\widehat{X}$ may be constructed in a simpler way, as a subset of $X \times \mathbb{R}$. Consider a simple example of $X=\mathbb{R}^{2}$ and the dynamical system $\pi_{0}(t,(x, y))=(t+x, y)$ with $M=\{0\} \times \mathbb{R}$ and $I(0, y)=(2, y)$. Then we can identify $M^{+}$with $M \times\{1\}$ and $M^{-}$with $M \times\{-1\}$. We can take $U^{+}=(0,1) \times\{0\}, U^{-}=(-1,0) \times\{0\}$ and "sink" and "lift" them respectively, to get $\widehat{X}$ homeomorphic to a disjoint union of two half-planes. However, this procedure is not applicable in a general situation.

REMARK. If there are no stationary points in $\left(X, \pi_{0}\right)$, the reasoning from Step 13 may be simplified, with the use of the equality $\widetilde{\chi}(\alpha+\beta, x)=$ $\tilde{\chi}(\beta, z)+\widetilde{\chi}\left(\alpha, \pi_{0}(\beta, x)\right)$ (cf. Remark following Lemma 2).

Remark. Decomposition dynamics, i.e. decompositions of the phase space with respect to equivalence classes carrying some dynamical structure, is an important chapter of the theory of dynamical systems. Some decompositions are taken with respect to the equivalence classes of a recurrence relation (see, for example, $[\mathrm{G} 1],[\mathrm{Hu}],[\mathrm{R}]$ ). Also, remetrizations of the phase space to get a system with the desired properties have been considered (see [BG], [G2]). However, in this paper decompositions as well as changes of topology are used in a different way.

From Theorem 1 and [MC, Th. 4.1] we have an immediate

Corollary 3. Let $X$ be a locally compact separable metric space and $\left(X, \pi_{0}, M, I\right)$ be an impulsive system given by a semidynamical system $\left(X, \pi_{0}\right)$. Assume that assumptions (1)-(3) of Theorem 1 hold. Then $\left(X, \pi_{0}\right)$ is isomorphic to a semidynamical system $\left(X, \pi_{1}\right)$ such that in the impulsive system $\left(X, \pi_{1}, M, I\right)$ we have $\widetilde{\omega}_{1}(x)=+\infty$ for any $x \in X$.

In [K3] and [K4] Kaul investigates several properties of impulsive semidynamical systems. He considers systems on a metric space $X$ where

(i) $\Omega$ is an open subset of $X$, the impulse set $M$ is the boundary of $\Omega$ and $I: M \rightarrow \Omega$,

(ii) $\phi$ is continuous in $\Omega$,

(iii) for any $x \in X$ we have $\widetilde{\omega}(x)=+\infty$.

If $M=\operatorname{Fr} \Omega$ it is natural to require (STC). When (STC) holds, assumption (ii) is satisfied, according to [K1] and [C2]. We have $M \cap I(M)=\emptyset$, as $I(M) \subset \Omega$. So, for $I$ closed we may apply Theorem 1 . Thus we have

COROllary 4. Suppose an impulsive dynamical systems satisfies the above condition (i). Then the system is isomorphic (the isomorphism being only a time reparametrization) to a system for which the results proved in [K3] and [K4] hold, provided that (STC) holds and I is closed. 
REMARK. Note that some results of [K3] and [K4] do not involve time, they only describe the properties of sets defined in such systems (for instance [K3, Th. 3.5]). Therefore, by the above theorems, those results are true under the assumptions of Corollary 3.4 without assuming (iii) because the relevant isomorphism only changes the speed of motion and not the trajectories as sets.

REMARK. Notice that the stability investigated in [K3] and [K4] is completely different from that considered in [C3].

EXAmple 1. Consider an impulsive system $\left(\mathbb{R}, \pi_{0}, M, I\right)$ given by the dynamical system $\pi_{0}(t, x)=t+x$. We put $M=\mathbb{N}$ and $I(n)=n+1-$ $1 /(n+2)^{2}$ (see Figure 1). Here $\widetilde{\omega}_{0}(x)<+\infty$ for any $x$. In particular, for $x<0$ we have $\widetilde{\omega}_{0}(x)=-x+\sum_{n=2}^{\infty} 1 / n^{2}$.

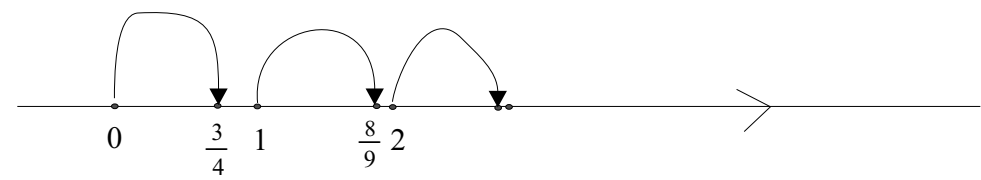

Fig. 1

The local semidynamical system obtained, according to Theorem 2, from this impulsive system may be described as follows (for convenience, we consider the phase space $Y$ homeomorphic to $\widetilde{\mathbb{R}}$; see Figure 2):

$$
Y=((-\infty, \alpha) \times\{0\}) \cup \bigcup\left\{\left\{p_{k}\right\} \times\left[0, \beta_{k}\right]: k=2,3,4, \ldots\right\} \subset \mathbb{R}^{2}
$$

(with euclidean topology), where

$$
\begin{gathered}
\alpha=\sum_{n=2}^{\infty} \frac{1}{n^{2}}, \quad p_{k}=\frac{1}{2^{2}}+\cdots+\frac{1}{k^{2}}, \quad \beta_{k}=1-\frac{1}{k^{2}} ; \\
\widetilde{\pi}_{0}(t,(x, 0))=(t+x, 0) \quad \text { for } t+x \leq \alpha, \\
\widetilde{\pi}_{0}\left(t,\left(p_{k}, \beta\right)\right)= \begin{cases}\left(p_{k}, \beta-t\right) \quad \text { for } t \leq \beta, \\
\left(p_{k}+t-\beta, 0\right) & \text { for } \beta \leq t<\beta+\alpha-p_{k} .\end{cases}
\end{gathered}
$$

Removing all the points $\left(p_{k}, \beta_{k}\right)$ we get a semidynamical system without start points.

After a suitable isomorphism we can get a system $\left(\mathbb{R}, \pi_{1}\right)$, where $\widetilde{\omega}_{1}(x)=$ $+\infty$ for each $x$. For instance, we can obtain a system $\pi_{1}$ which is defined as follows.

First define a sequence $\left(a_{n}\right)$ by $a_{2 k}=k, a_{2 k+1}=(k+1)-1 /(k+2)^{2}$. Then put

$$
\begin{aligned}
\pi_{1}(t, x) & =t+x & & \text { if } x \leq 0, t+x \leq 0 \\
\pi_{1}\left(t, a_{n}\right) & =a_{n}+\left(a_{n+1}-a_{n}\right) t & & \text { if } t \in[0,1], n \in \mathbb{N} .
\end{aligned}
$$




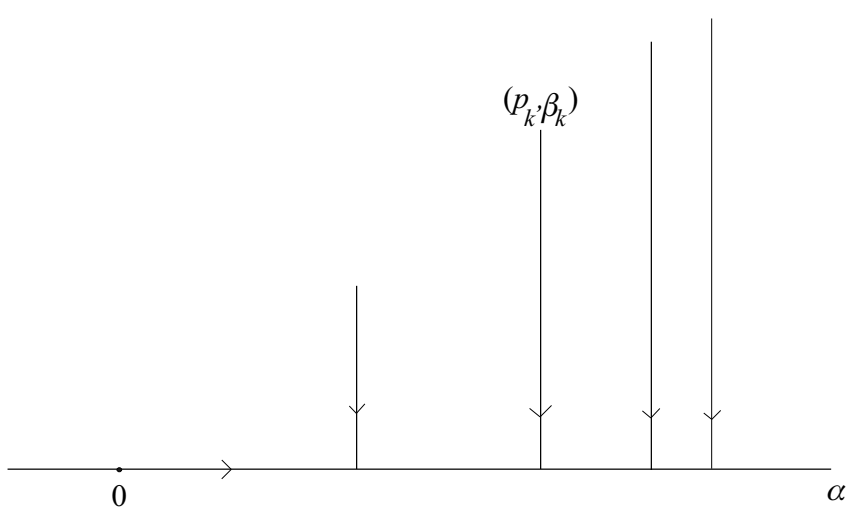

Fig. 2

This determines $\pi_{1}(t, x)$ in a unique way for any $t, x \in \mathbb{R}$. The system $\left(\mathbb{R}, \pi_{1}\right)$ is isomorphic to $\left(\mathbb{R}, \pi_{0}\right)$, and $\left(\mathbb{R}, \pi_{1}, \mathbb{N}, I\right)$ satisfies the assertion of Theorem 1 .

The system $\left(\mathbb{R}, \pi_{1}\right)$ gives a global semidynamical system $\left(Z, \widetilde{\pi}_{1}\right)$ (also after a suitable homeomorphism of the space; see Figure 3):

$$
\begin{aligned}
Z= & (\mathbb{R} \times\{0\}) \cup(\{k\} \times[0,1]: k=1,2,3, \ldots\} \subset \mathbb{R}^{2}, \\
& \tilde{\pi}_{1}(t,(x, 0))=(t+x, 0), \\
& \tilde{\pi}_{1}(t,(k, \beta))= \begin{cases}(k, \beta-t) & \text { for } t \leq \beta, \\
(k+t-\beta, 0) & \text { for } t \geq \beta .\end{cases}
\end{aligned}
$$

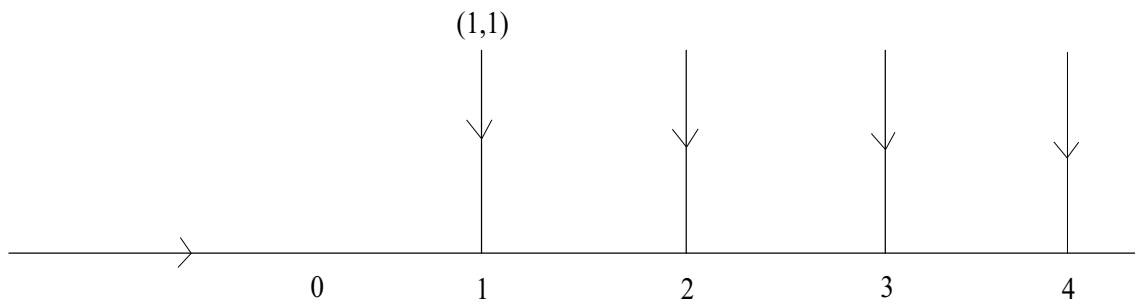

Fig. 3

Example 2. Consider an impulsive system $\left(\mathbb{R}^{2}, \pi_{0}, M, I\right)$ given by the dynamical system described by the differential equations $\theta^{\prime}=0, r^{\prime}=-r$ (in polar coordinates). Let $M=[0,2 \pi] \times\{1\}, I(\theta, 1)=(\theta+\alpha, 2)$, where $\alpha$ is given; here $I(M)=[0,2 \pi] \times\{2\}$ (Figure 4 ).

Then the (global) semidynamical system $\widetilde{\pi}_{0}$ obtained from $\pi_{0}$ may be described as a system on $Y=\mathbb{T} \cup A \cup B \subset \mathbb{R}^{3}$, where $\mathbb{T}$ is the torus obtained by rotating the circle $=\left\{(x, y, z):(x-3 / 4)^{2}+z^{2}=1 / 16, y=0\right\}$ about the line $\{(x, y, z): x=0, y=0\}$ (we have $\mathbb{T} \cap\left(\mathbb{R}^{2} \times\{0\}\right)=\left\{(x, y, z): x^{2}+y^{2}=1\right.$ or $\left.\left.x^{2}+y^{2}=1 / 4, z=0\right\}\right)$, and

$$
A=\left\{(x, y, z): x^{2}+y^{2} \geq 1, z=0\right\}, \quad B=\left\{(x, y, z): x^{2}+y^{2} \leq 1, z=2\right\} .
$$




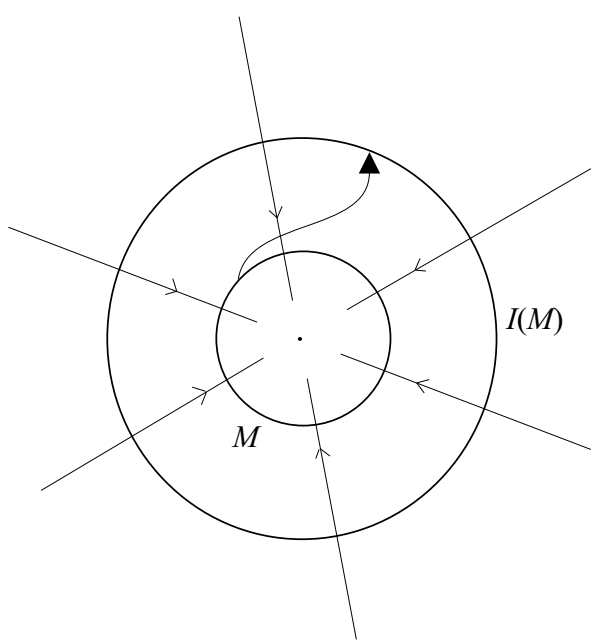

Fig. 4

The set $Y$ is disconnected, it has two components: $\mathbb{T} \cup A$ and $B$. The motion on $B$ does not give anything new, each trajectory converges to the stationary point 0 . The trajectory of any $p \in A$ goes along a straight line and after some time joins $\mathbb{T}$ where it starts behaving according to the law of motion on $\mathbb{T}$. A feature of this example is the new motion on $\mathbb{T}$. Here we have a dynamical system on the torus. Its trajectories depend on $\alpha$. If $\alpha / 2 \pi \in \mathbb{Q}$, then all the trajectories are periodic (in particular, for $\alpha=0$ each trajectory is a circle). If $\alpha / 2 \pi \notin \mathbb{Q}$, then the trajectory of any $p \in \mathbb{T}$ is dense in $\mathbb{T}$.

ExAmple 3. Consider an impulsive system $(X, \pi, M, I)$ given by the dynamical system on $\mathbb{R} \times(-1,1)$ described by the differential equations $y^{\prime}=0, x^{\prime}=e^{1+1 /(|y|-1)}$. Let $M=\{0\} \times(-1,1)$ and $I(x, y)=(1,0)$ for each $(x, y) \in M$ (see Figure 5).

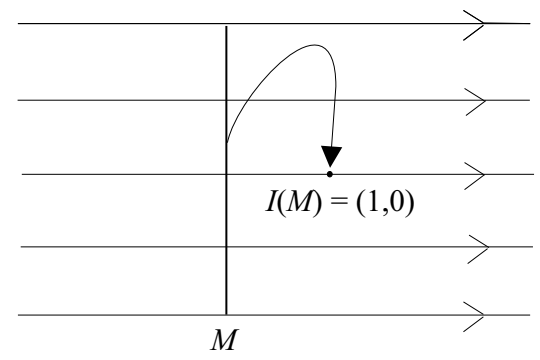

Fig. 5

Theorem 2 yields a semidynamical system $(\widetilde{X}, \widetilde{\pi})$. The phase space $\widetilde{X}$ is not metrizable. It can be easily verified that it has no countable basis at $(1,0)$. One can also see that assertion (a) of Theorem 2 is not satisfied as 
$I^{-1}((1,0))$ is not compact. However, one could glue all the points of $M$ with $(1,0)$ and introduce the topology in the new space in another natural way to get a metric space. We may transform the topology with the use of vertical segments $\{x\} \times(-1,1)$ for $x<0$. Then the new space $\breve{X}$ may be described (after a suitable homeomorphism $f$ ) as $\breve{X}=[0,+\infty) \times(-1,1) \times\{0\} \cup Z$, where $Z=\left\{(x, y, z) \in \mathbb{R}^{3}: x=1, z>|y|\right\}$ with euclidean topology (we get this by transforming any segment $\{(x, y): x=-\alpha, y \in(-1,1)\}$ linearly onto a segment $\{(x, y, z): x=1, z=\alpha,|y|<z\}$ ) (see Figure 6$)$. In the new system, for any $y$ the point $(0, y, 0)$ is a start point.

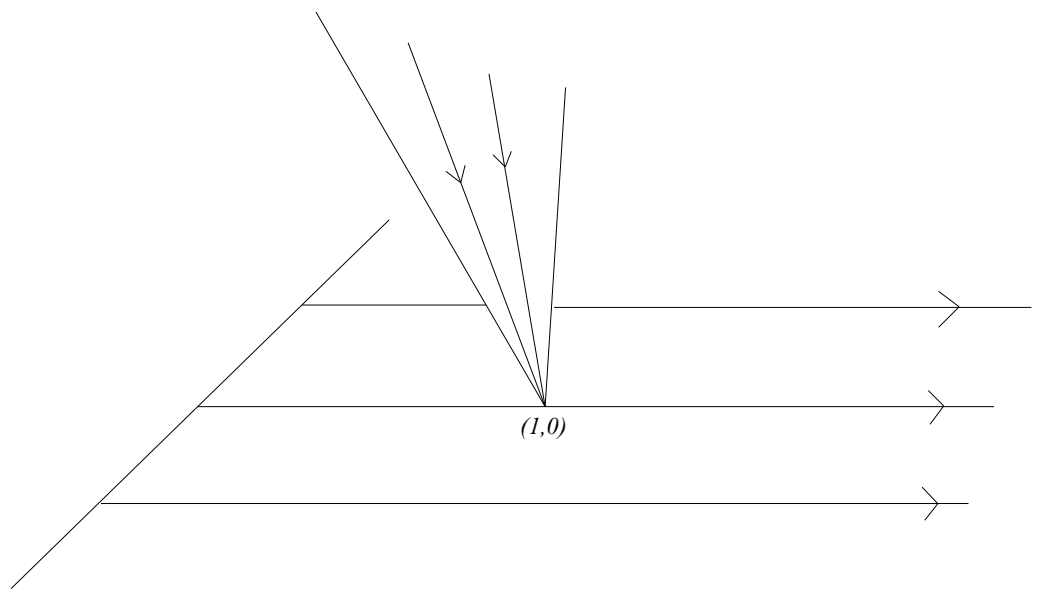

Fig. 6

Obviously the resulting space is metric. However, after glueing we cannot get a semidynamical system, as the function $\breve{\pi}$ obtained is not continuous! We can only get the continuity of the motion $\breve{\pi}(\cdot, p)$ for the given $p$.

To show the discontinuity of $\breve{\pi}$, consider $p_{n}=(-1 / n, 1-1 / n) \in X$ and $t=1$. After the transformation $f$ of $(-\infty, 0) \times(-1,1)$ onto $Z$ we have $f\left(p_{n}\right)=q_{n}$, where $q_{n}=\left(1, \beta_{n}, 1 / n\right)$ with $\beta_{n}<1 / n$. Hence $q_{n} \rightarrow(1,0,0)$. Notice that $\breve{\pi}\left(1, q_{n}\right) \in Z$ for $n>1$, as $e^{1+1 /(1-1 / n-1)}<1 / n$ and $\pi\left(1, p_{n}\right) \in$ $(-1 / n, 0) \times(-1,1)$ (it does not reach $M)$. It follows that $\breve{\pi}\left(1, q_{n}\right) \rightarrow(1,0,0)$. On the other hand, $\breve{\pi}(1,(1,0,0)) \neq(1,0,0)$, so that $\breve{\pi}\left(1, q_{n}\right) \nrightarrow \breve{\pi}(1,(1,0,0))$. Therefore the constructed system is pseudo-semidynamical, but not semidynamical.

\section{References}

[AV] J. M. Aarts and J. de Vries, Local dynamical systems and their morphisms, Math. Centre Tracts 115 (1979), 1-14.

[BH] N. P. Bhatia and O. Hajek, Local Semi-Dynamical Systems, Lecture Notes in Math. 90, Springer, 1970. 
[BS] N. P. Bhatia and G. P. Szegö, Stability Theory of Dynamical Systems, Springer, 2002.

[BG] A. Bosznay and B. Garay, Remetrization and a new type of recurrence, Funkcial. Ekvac. 30 (1987), 57-68.

[Ca] D. H. Carlson, A generalization of Vinograd's Theorem for dynamical systems, J. Differential Equations 11 (1972), 193-201.

[CV] C. O. Christenson and W. L. Voxman, Aspects of Topology, Dekker, 1977.

[C1] K. Ciesielski, Continuity in semidynamical systems, Ann. Polon. Math. 46 (1985), 61-70.

[C2] - On semicontinuity in impulsive dynamical systems, Bull. Polish Acad. Sci. Math. 52 (2004), 71-80.

[C3] - On stability in impulsive dynamical systems, ibid. 52 (2004), 81-91.

[C4] - Sections in semidynamical systems, ibid. 40 (1992), 297-307.

[CO] K. Ciesielski and K. Omiljanowski, Topological description of cuts and sections in semiflows on 2-manifolds, Topology Appl. 60 (1994), 13-22.

[E1] S. Elaydi, Semidynamical systems with nonunique global backward extensions, Funkcial. Ekvac. 26 (1983), 173-187.

[E2] - Semidynamical systems with nonunique global backward extensions II: the negative aspects, ibid. 27 (1984), 85-100.

[EK] S. Elaydi and S. Kaul, Semiflows with global extensions, Nonlinear Anal. 10 (1986), 713-726.

[E] R. Engelking, General Topology, Polish Sci. Publ., 1977.

[G1] B. Garay, Uniform persistence and chain recurrence, J. Math. Anal. Appl. 139 (1989), 372-381.

[G2] - , Auslander recurrence and metrization via Liapunov functions, Funkcial. Ekvac. 28 (1985), 299-308.

[Ha] O. Hajek, Dynamical Systems in the Plane, Academic Press, 1968.

$[\mathrm{Hu}] \quad \mathrm{M}$. Hurley, Chain recurrence, semiflows and gradients, J. Dynam. Differential Equations 7 (1995), 437-456.

[K1] S. Kaul, On impulsive semidynamical systems, J. Math. Anal. Appl. 150 (1990), 120-128.

[K2] - On impulsive semidynamical systems II. Recursive properties, Nonlinear Anal. 16 (1991), 635-645.

[K3] - Stability and asymptotic stability in impulsive semidynamical systems, J. Appl. Math. Stochastic Anal. 7 (1994), 509-523.

[K4] - Stability and attraction in impulsive semidynamical systems, in: World Congress of Nonlinear Analysts '92, de Gruyter, 1996, 1349-1356.

[Ku] K. Kuratowski, Topology, Vol. I, Academic Press and Polish Sci. Publ., 1966.

[LBS] V. Lakshmikantham, D. D. Bainov and P. S. Simeonov, Theory of Impulsive Differential Equations, World Sci., 1989.

[MC] R. C. McCann, Negative escape time in semidynamical systems, Funkcial. Ekvac. 20 (1977), 39-47.

[N] J. Nagata, A theorem for metrizability of a topological space, Proc. Japan Acad. Sci. 33 (1957), 128-130.

[NS] V. V. Nemytskii and V. V. Stepanov, Qualitative Theory of Differential Equations, Princeton Univ. Press, 1960.

[P1] A. Pelczar, General Dynamical Systems, Jagiellonian Univ. Lecture Notes 293, 1978 (in Polish).

[P2] - Introduction to the Theory of Differential Equations, part II, Math. Library 67, Polish Sci. Publ., 1989 (in Polish). 
[R] C. Robinson, Dynamical Systems: Stability, Symbolic Dynamics, and Chaos, CRC Press, 1995.

[Sa] S. H. Saperstone, Semidynamical Systems in Infinite Dimensional Spaces, Springer, 1981.

[U1] T. Ura, Isomorphisms and local characterization of local dynamical systems, Funkcial. Ekvac. 12 (1969), 99-122.

[U2] - Local dynamical systems and their isomorphisms, in: Lecture Notes in Math. 243, Springer, 1971, 76-90.

[U3] - Local isomorphisms and local parallelizability in dynamical systems theory, Math. Systems Theory 3 (1969), 1-16.

[UE] T. Ura and J. Egawa, Isomorphisms and parallelizability in dynamical systems theory, ibid. 7 (1973), 250-264.

[V] J. de Vries, Elements of Topological Dynamics, Math. Appl. 257, Kluwer, 1993.

Mathematics Institute

Jagiellonian University

Reymonta 4

30-059 Kraków, Poland

E-mail: ciesiels@im.uj.edu.pl

Reçu par la Rédaction le 31.10.2003

Révisé le 7.9.2004 